\title{
Micosis cutáneas prevalentes en la infancia
}

\author{
Cutaneous mycoses prevalent in childhood
}

\author{
Ana Paula Giraldo-Hoyos ${ }^{1}$, Nora Cardona-Castro ${ }^{2}$ \\ 1. Médica, Residente de Dermatología, Universidad CES, Medellín, Colombia \\ 2. Médica M.Sc. Universidad CES, Instituto Colombiano de Medicina Tropical, Medellín, Colombia
}

\section{Resumen}

Las micosis cutáneas son un grupo de entidades que se presentan con gran frecuencia en las consultas dermatológicas y, aunque la principal población afectada son adolescentes y adultos, en la población pediátrica se ha incrementado dicha incidencia debido a múltiples factores que han hecho más frecuente el contagio persona-persona y por otras fuentes. Por tal razón, es importante tener en cuenta a la hora de evaluar un paciente en este grupo etario, las principales micosis cutáneas, su diagnóstico y manejo, para de esta manera, evitar manejos fallidos y resultados desfavorables.

PALABRAS CLAVE: micosis cutáneas, infancia, dermatofitos

\author{
Correspondencia: \\ Ana Paula Giraldo \\ Email: \\ paula88_2@hotmail.com
}

Recibido 19 de junio de 2014.

Aceptado: 25 de julio de 2014.

No se reportan conflictos de interés.

\section{Summary}

Cutaneous mycoses is a group of entities that occur with high frequency in dermatology consultations, and although the main affected population are adolescents and adults, the incidence in the pediatric population has increased due to multiple factors like person to person transmission and other sources. For this reason it is important to consider major cutaneous mycoses when evaluating a patient in this age group, its diagnosis and management, and in this way, to avoid handling failures and unfavorable results.

KEY WORDS: Cutaneous mycoses, childhood, dermatophytes.

\section{Introducción}

Las micosis cutáneas son infecciones de la piel del ser humano, ya sea por disminución de las defensas del huésped o por la capacidad inherente del agente para causar invasión y, finalmente, un proceso infeccioso.

Pocas especies de hongos son patógenas para el humano, pero las micosis hacen parte importante de las consultas dermatológicas ${ }^{1}$. Actualmente, con el avance en el uso de medicamentos inmunosupresores, el contacto estrecho entre niños en las guarderías y el aumento en la frecuencia de viajes, ciertas entidades, entre ellas las micosis, se han hecho más prevalentes ${ }^{1-3}$.

En la población pediátrica, la frecuencia de micosis cutáneas no son una de las principales causas de morbimortalidad, y representan tan solo entre el 8 y el $10 \%$ de las dermatosis en niños ${ }^{1}$; no obstante, son importantes ya que debemos reconocerlas a tiempo para darles el tratamiento adecuado y así evitar complicaciones y transmisión a otros niños. Por tal motivo, la prevalencia e incidencia de esta enfermedad en los niños ha sido poco estudiada, con sólo algunos reportes en América Latina y la mayoría en países desarrollados, donde su incidencia es aún más baja ${ }^{4}$.

\section{Agentes causales}

Los dermatofitos son hongos queratinofílicos, con capacidad de invadir el estrato córneo y otros tejidos que- 
ratinizados, como uñas y pelo ${ }^{5,6}$. Según su hábitat, se clasifican en antropofílicos, geofílicos y zoofílicos. Para los antropofílicos, el ser humano es el huésped definitivo y la especie más frecuente es Trichophyton rubrum; para los geofílicos, los suelos son el hábitat natural y la especie más frecuente es Microsporum gypseum; y los zoofílicos habitan en los animales y se transmiten al humano de manera accidental por medio del contacto; la especie más importante es Microsporum canis.

Cabe resaltar que, de todos estos, los que mayor respuesta inflamatoria inducen son los dermatofitos zoofílicos, ya que usan la queratina de una manera menos especializada que los antropofílicos y causan una mayor reacción inmunitaria ${ }^{4-6}$.

En Latinoamérica y México, los cinco dermatofitos más frecuentes en la población general son $T$. rubrum (70 \%), M. canis (13\%), T. mentagrophytes (10\%), T. tonsurans (3\%) y Epidermophyton flocosum (1\%).

Las dermatofitosis también se clasifican según su ubicación corporal, y se denominan tiña de la cabeza, de la barba, del cuerpo, de la región inguinal, de las manos y de los pies. Entre las tiñas, o dermatofitosis, la más frecuente en la población pediátrica es la tiña de la cabeza, seguida por la del cuerpo y finalmente, en los últimos años se ha visto un incremento en la tiña de las uñas, ya que las demás, como tiña de manos, pies y barba son muy raras en niños ${ }^{1-3}$.

\section{Tiña de la cabeza (tinea capitis)}

Es la dermatofitosis más común en la infancia ${ }^{6}$. Sus factores predisponentes son un bajo nivel socioeconómico, falta de condiciones higiénicas, humedad, estados de desnutrición y contacto estrecho entre niños en guarderías y escuelas. Su incidencia se encuentra en aumento ${ }^{6-10}$, principalmente en países en desarrollo y en algunos lugares de Europa, como España, Francia, Italia y Eslovenia ${ }^{6,9-12}$

Sus principales agentes causales son los géneros $\mathrm{Mi}$ crosporum y Trichophyton ${ }^{2,10}$, de los cuales el agente más prevalente es $M$. canis, aunque se han encontrado variaciones con el tiempo y el área geográfica ${ }^{6,10,13}$. En Norteamérica y el Reino Unido, el agente causal en el $90 \%$ de casos es $T$. tonsurans, mientras que en los países en desarrollo, como Perú, Brasil, México, Puerto Rico y Venezuela, y en países desarrollados de Europa Central y el Mediterráneo, $M$. canis es el agente predominante, aunque en dichos países la tiña de la cabeza por T. tonsurans se encuentra en aumento en los últimos años, como también sucede en Colombia ${ }^{14}$. Las únicas especies de dermatofitos que no producen tinea capitis son E. floccosum y T. concentricum, mientras que T. rubrum, que es el dermatofito más aislado en todo el mundo, puede producirla pero en raras ocasiones ${ }^{6}$.

La prevalencia de tiña de la cabeza se estima en 3 a 8\% de la población pediátrica en Estados unidos. En Colombia, los datos epidemiológicos hablan de cerca de $4,8 \% \%^{15}$, mientras que en España sólo se encuentra en $0,33 \%$ y en Londres, en $2,5 \% \%^{5,10,16}$, aunque en muchos lugares del mundo este dato estadístico aún permanece desconocido ${ }^{6,16,17}$.

El agente etiológico puede variar según el sexo, encontrándose más frecuentemente afectado el sexo masculino por $M$. canis, mientras que T. tonsurans afecta ambos sexos por igual ${ }^{5-7,10,18}$. Existen variaciones con respecto al sexo más afectado, pero en la mayoría de los estudios se reporta una mayor frecuencia en el sexo masculino.

La razón que se ha planteado para que la entidad se presente más frecuentemente en hombres, tiene que ver con el influjo hormonal en la prepubertad, ya que en esta época los niños tienen menor cantidad de hormonas sexuales que las niñas y los dermatofitos característicamente poseen proteínas citosólicas que se unen específicamente a la progesterona y otras hormonas (estradiol, testosterona) y estas inhiben el crecimiento fúngico; esto hace que las infecciones sean menos comunes en las mujeres, ya que se retarda el crecimiento de las hifas, permitiendo al sistema inmunitario responder más eficazmente. También se ha encontrado asociación con el nivel de ácidos grasos fungistáticos que se encuentran a más temprana edad en el sexo femenino ${ }^{6,19}$. Por otro lado, el sexo masculino tiene mayor contacto con animales por trabajar más frecuentemente en zonas rurales y tienen el pelo más corto, lo que facilita la visualización de las lesiones ${ }^{10}$.

La edad promedio de presentación es entre los 3 y los 7 años de edad ${ }^{5,6,10}$, con un rango entre los 2 y los 12 años según la población estudiada ${ }^{1-4}$, aunque puede verse también en menores de $1 \mathrm{año}^{10,20,21}$. Cuando se presenta en adultos, son generalmente mujeres con trastornos hormonales, inmunosupresión por enfermedades linfoproliferativas o medicamentos ${ }^{6}$.

Aunque la tiña de la cabeza no es una condición que amenace la vida, continúa siendo un factor de morbilidad importante, tanto a nivel físico como social y económico ${ }^{7,16}$.

La clasificación de esta entidad es compleja, y se hace según el patrón microscópico de invasión fúngica en endothrix y ecto-endothrix y según su presentación clínica.

Según el patrón de invasión fúngica, la forma endothrix tiene dos variedades: tricofítica y fávica ${ }^{6}$, y es producida principalmente por el género Trichophyton y con mayor frecuencia, T. tonsurans. En la va- 
riedad tricofítica, las hifas crecen por dentro del folículo y penetran la vaina del pelo, mientras que la cutícula permanece intacta 6 . El favus por su parte, es causado por $T$. shoenleinii y produce un tipo de tiña inflamatoria que deja característicamente múltiples costras descamativas en forma de casco o "godete fávico" alrededor de los pelos ${ }^{6}$. La invasión de tipo ectoendothrix tiene tres variedades y se asocia generalmente con especies de microsporum; de ellas, las más importantes son M. canis, M. audouinii, M. gypseum y M. ferrugineum. En este tipo de invasión, las hifas se encuentran en la porción media del folículo piloso y cubren el pelo en la superficie, produciendo destrucción de la cutícula y crecimiento alrededor de la vaina externa del pelo ${ }^{6}$.

\section{Presentación clínica}

Hasta el momento se han descrito una amplia gama de presentaciones que van desde un estado de portador asintomático a formas no inflamatorias e inflamatorias, todo esto según el origen del agente causal (zoofílico, geofílico o antropofílico) y del estado inmunitario del huésped ${ }^{6}$. El tipo de presentación no necesariamente indica el agente causal, ya que, así como han cambiado los agentes más importantes según el área geográfica, también han variado los agentes según la presentación clínica; es decir, las variedades tricofíticas pueden ser causadas por especies de Microsporum, mientras que las microspóricas pueden estar relacionadas con Trichophyton ${ }^{6}$.

\section{Variedad no inflamatoria o seca}

Se caracteriza por descamación, pelos cortos, gruesos y quebradizos de dos a tres milímetros en el área afectada, con una vaina blanquecina. Hay dos variedades de presentación, según el agente causal y sus manifestaciones: tricofítica y microspórica. La variedad tricofítica o "de los puntos negros", se caracteriza por varias placas de tamaño pequeño, con algunos pelos más cortos que otros (alopecia irregular) y que por estar fracturados a la altura del ostium folicular, dan la apariencia de puntos negros ${ }^{6}$. El principal agente etiológico de este grupo es T. tonsurans ${ }^{2,22}$. En la variedad microspórica se observan por lo general, una o escasas placas redondas de mayor tamaño, con los pelos fracturados a la misma altura, generalmente más arriba de la salida del ostium folicular y son asintomáticas o producen mínimo prurito ${ }^{2,6,22}$.

\section{Tiña inflamatoria de la cabeza}

Esta forma de presentación se divide en dos variedades: el querion de Celso y la tiña fávica.

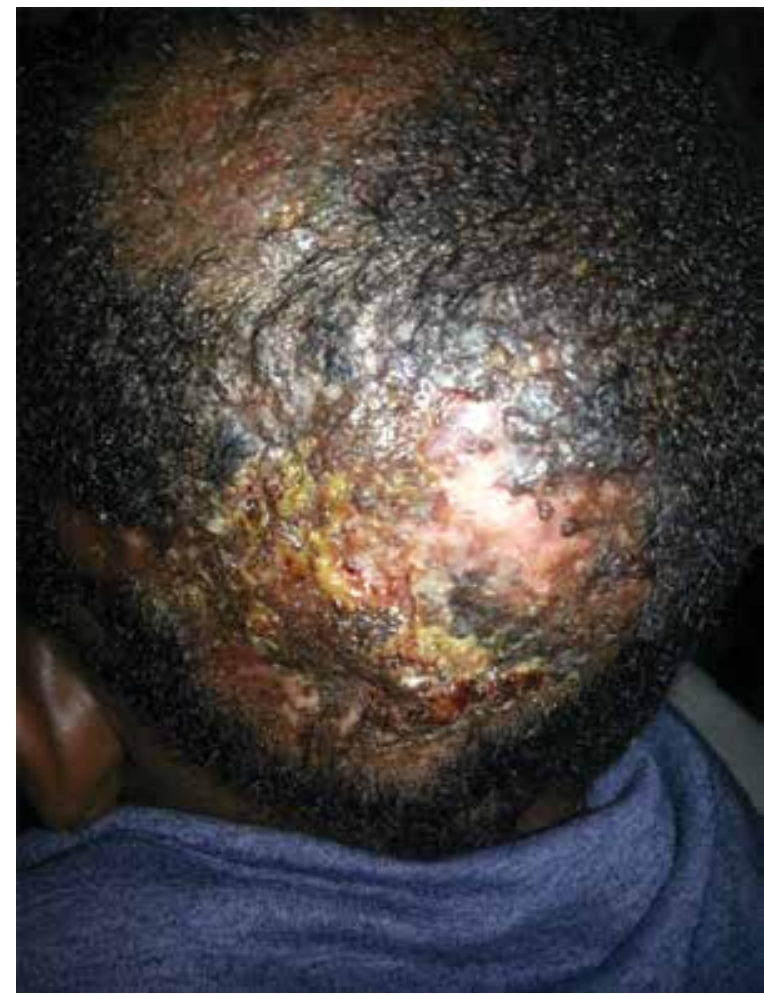

FigurA 1. Paciente de 4 años de edad con querion de Celso: placa descamativa con costras, múltiples pústulas y secreción purulenta.

La principal o más común es llamada querion de Celso y se caracteriza por un nódulo inflamatorio generalmente único, de tamaño mediano a grande y muy doloroso a la palpación. Se inicia como una tiña no inflamatoria, con una o varias placas seudoalopécicas descamativas y pelos cortos, pero posteriormente se torna inflamatoria, eritematosa y levantada, y presenta múltiples pústulas por las que drena material purulento (FIGURA 1). El síntoma más importante es el dolor, y en esta fase se presentan adenopatías retroauriculares y satélites. Puede presentarse también malestar general y fiebre 5 .

Si el proceso continúa, en aproximadamente ocho semanas la respuesta inmunitaria del huésped resuelve por completo la infección, pero característicamente puede dejar como secuela alopecia cicatricial; por lo tanto, el diagnóstico debe llevarse a cabo de forma temprana ${ }^{2,6,22}$.

El querion de Celso puede ser producido por diferentes especies de dermatofitos, pero generalmente se aíslan $M$. canis, $M$. gypseum, T verrucosum y T. tonsurans ${ }^{23}$.

La segunda forma de tiña inflamatoria es el favus o tiña fávica, una infección causada principalmente por T. schoenleinii y, con menos frecuencia, por M. gypseum y T. violaceum ${ }^{16,24}$. Clínicamente, es una tiña in- 
flamatoria que produce costras amarillas elevadas en forma de godete o escudo alrededor de los folículos; además, se caracteriza por presentar un olor característico a "ratón" o "queso". En las costras hay abundantes hifas que son muy infecciosas ${ }^{5,6}$.

Otros posibles hallazgos en la tiña de la cabeza son las reacciones 'ide' ${ }^{9}$ y las lesiones de eritema nudoso, que pueden presentarse con la entidad o con el inicio del tratamiento antifúngico, pero que no deben ser motivo de suspensión de este ${ }^{25}$ También puede haber diseminación de la infección, dando lugar a otras tiñas, entre ellas, del cuerpo, manos, pies y uñas ${ }^{5,26}$; además las lesiones pueden sobreinfectarse por Staphylococcus aureus $^{27}$. En ocasiones, se pueden encontrar estados de portador, principalmente en adultos, y que rara vez presentarán manifestaciones clínicas, pero sirven como fuente de contagio para niños mediante el contacto directo o por medio de objetos de uso en común, como cepillos, almohadas, muebles, juguetes y auriculares, donde las estructuras fúngicas pueden vivir por algunos meses $^{16,28-30}$. Se han encontrado también casos de portadores asintomáticos en niños de raza negra, principalmente en Estados Unidos y Francia ${ }^{31}$.

\section{Diagnóstico}

El diagnóstico de esta entidad es clínico y de laboratorio. La presencia de placas alopécicas con descamación, inflamación perifolicular y adenopatías regionales, debe hacer sospechar la presencia de tiña de la cabeza. La luz de Wood es una fácil de realizar en el consultorio y proporciona una herramienta útil, ya que se puede observar una fluorescencia amarillo-verdosa característica en la mitad de los casos de las tiñas microspóricas o ecto-endothrix ${ }^{32}$, mientras que, en las variedades tricofíticas esta es negativa ${ }^{6,33}$.

Se debe tomar muestra de los pelos cortos o puntos negros para el estudio fúngico, teniendo en cuenta que se debe hacer limpieza previa de la zona con alcohol al $70 \%$ para evitar la contaminación bacteriana ${ }^{5,34}$.

El examen directo se hace con $\mathrm{KOH}$ al 10-30 \% más dimetilsulfóxido o negro de clorazol, en busca de hifas o esporas; posteriormente, se hace el cultivo de la muestra en agar Sabouraud o agar Mycosel ${ }^{\mathrm{TM}}$ y se incuba entre 25 y $30{ }^{\circ} \mathrm{C}$, obteniéndose crecimiento de las colonias en una a seis semanas; no obstante, pueden haber falsos negativos hasta en $50 \%$ de las muestras ${ }^{6,34}$.

Existe un estudio inmunológico en el cual se emplea la tricofitina para evaluar la reacción inmunitaria del huésped. Este antígeno se extrae de T. mentagrophytes, que presenta reacción cruzada con los demás dermatofitos y produce dos respuestas, una inmediata (de tipo I) y otra tardía (de tipo IV); la primera es posi- tiva en casos crónicos o en pacientes atópicos y la segunda, en casos agudos como el querion de Celso ${ }^{6}$.

El estudio histopatológico puede demostrar la presencia de las artrosporas dentro de los folículos o en el estrato córneo; también se encuentra atrofia de los folículos y un infiltrado inflamatorio en la dermis. En el caso del querion de Celso, los hallazgos pueden clasificarse de acuerdo con el patrón inflamatorio ${ }^{35}$.

\section{Diagnóstico diferencial}

En el diagnóstico diferencial de la tiña de la cabeza se deben considerar múltiples entidades que tienen gran similitud clínica, como alopecia areata, foliculitis bacteriana, dermatitis seborreica, dermatitis atópica, psoriasis, tricotilomanía y tricorrexis nudosa ${ }^{2,6,16,24}$.

\section{Tratamiento}

Con este se busca curación clínica y de laboratorio en el menor tiempo posible, con la intención de evitar secuelas, principalmente en el caso de las tiñas inflamatorias. Tanto en las formas inflamatorias como en las no inflamatorias son útiles los mismos medicamentos, pero además, en las formas inflamatorias se puede considerar el uso de ciclos cortos de esteroides sistémicos por una a dos semanas (prednisona a dosis de $1 \mathrm{mg}$ / $\mathrm{kg}$ al día) o su aplicación intralesional ${ }^{6,36,37}$.

\section{Tratamiento tópico}

Sólo se usa en lactantes con enfermedad de corta duración, ya que en la mayoría de los casos el medicamento no penetra adecuadamente el folículo piloso. Los champú con sulfuro de selenio al $2 \%$ o con ketoconazol al $2 \%$ se han usado tres veces a la semana por cinco minutos de contacto, con el fin disminuir las tasas de transmisión a otros niños, pero siempre en combinación con tratamiento sistémico ${ }^{6,38,39}$.

\section{Tratamiento sistémico}

Griseofulvina: Desde hace muchos años la griseofulvina ha sido el tratamiento de elección para la tiña de la cabeza y aún hoy sigue siendo la piedra angular en su manejo $0^{1,2,6,16,22}$. Este medicamento fungistático inhibe la síntesis de ácidos nucleicos e interrumpe la división celular en metafase, impidiendo así la síntesis de pared celular, además de tener acción antiinflamatoria. Comercialmente se consigue en tabletas de $500 \mathrm{mg}$. La dosis recomendada se calcula dependiendo de la forma que se encuentre disponible, de la fórmula 'micronizada' se usan 15 a $20 \mathrm{mg} / \mathrm{kg}$ por día, mientras que de la fórmula 'ultramicronizada' se recomiendan $10 \mathrm{mg} /$ $\mathrm{kg}$ por día por 6 a 12 semanas o preferiblemente, hasta 
15 días después de la cura clínica y de laboratorio ${ }^{2,6,29,33}$.

Entre los principales efectos secundarios se encuentran náuseas y erupciones exantemáticas y está contraindicado su uso en el embarazo $0^{2,6,40}$. Cuando la duración del tratamiento excede las ocho semanas, se recomienda hacer pruebas de función renal, hepática y hemograma de manera periódica ${ }^{34}$.

Terbinafina. Es una alilamina fungicida ${ }^{41}$, cuyo mecanismo de acción se encuentra en la membrana celular y es el medicamento más usado para el manejo sistémico de las dermatofitosis, a excepción de la tiña de la cabeza, en la que sólo se usa cuando esté contraindicado el tratamiento con griseofulvina, ya que se necesitan dosis altas por períodos mayores a cuatro semanas $5,40,42,43$. Comercialmente se consigue en tabletas de $250 \mathrm{mg}$. La dosis recomendada se calcula según el peso $(3$ a $6 \mathrm{mg} / \mathrm{Kg}$ por día), pero para efectos prácticos, puede hacerse de la siguiente manera: pacientes con menos de $20 \mathrm{~kg}, 62,5$ $\mathrm{mg} /$ día; de 20 a $40 \mathrm{~kg}, 125 \mathrm{mg} /$ día y más de $40 \mathrm{~kg}, 250$ $\mathrm{mg} / \mathrm{dia}^{2,6,40,44,45}$. Los principales efectos secundarios son malestar gastrointestinal, cefalea, ageusia, alteraciones hepáticas reversibles y erupciones cutáneas, principalmente en las primeras cuatro semanas de iniciado el medicamento ${ }^{45}$. Se recomienda realizar hemograma, función hepática y renal, antes del inicio del tratamiento y mensualmente durante este ${ }^{46}$.

Itraconazol. Es un triazólico de uso oral con actividad fungistática y fungicida. Debe administrarse con alimentos o bebidas ácidas para mejorar su absorción. Actúa mediante la depleción del ergosterol, alterando la permeabilidad de la membrana celular. Alcanza altas concentraciones en tejidos queratinizados (uñas, pelo y piel), y además en tejido adiposo, mesenterio, endometrio y cuello uterino. La dosis recomendada es de 3 a $5 \mathrm{mg} / \mathrm{kg}$ al día por cuatro a seis semanas, pero la reacción depende del agente etiológico, ya que la tiña de la cabeza producida por $M$. canis puede requerir un tratamiento de mayor duración ${ }^{6,47,48}$. Entre los principales efectos adversos se encuentran las múltiples interacciones medicamentosas (por la inhibición de la enzima citocromo P450), diarrea, dolor abdominal, vómito, edema, hipertensión arterial, hipertrigliceridemia, hipopotasemia, neutropenia y reacciones dérmicas. Es categoría $C$ en el embarazo ${ }^{25}$. Se debe hacer seguimiento con hemograma, pruebas de función renal y hepática, antes del inicio y cada mes durante el tratamiento ${ }^{48}$.

Fluconazol. Es otro medicamento triazólico de uso oral, con amplio espectro de actividad frente a dermatofitos y especies de Candida. Es muy hidrosoluble, por lo que se absorbe de manera independiente al pH gástrico. $\mathrm{Al}$ tener baja unión a proteínas plasmáticas, alcanza altas concentraciones en piel, uñas, sudor, vagina, esputo, líquido peritoneal y orina. Actúa en los esteroles de la membrana celular como los demás azoles, pero con una afinidad mucho mayor ${ }^{5,6,25}$. La dosis recomendada es $6 \mathrm{mg} / \mathrm{kg}$ al día por 20 días aunque no es un medicamento de primera línea 49 .

\section{Tiña del cuerpo (tinea corporis)}

Es una dermatofitosis superficial que afecta la piel del cuerpo sin vello terminal (piel lampiña). Con frecuencia es causada por algunas especies de Microsporum y Trichophyton, entre las cuales las más comunes en nuestro medio son T. rubrum, T. mentagrophytes, $M$. canis y $M$. audouinii ${ }^{2,22}$.

Produce como lesiones características placas eritemato-descamativas pruriginosas ${ }^{22}$. Esta entidad puede presentarse a cualquier edad, y en la población pediátrica ocupa el segundo lugar de las micosis cutáneas después de la tiña de la cabeza. Se presenta principalmente en el grupo de los 2 a 10 años de edad, en ambos sexos por igual y más frecuentemente en climas húmedos tropicales. Según la especie de dermatofito y la reacción del huésped, pueden presentarse diferentes tipos de lesiones ${ }^{2}$. Puede afectar cualquier región corporal pero es más frecuente en tronco (50 \%), extremidades $(30 \%)$ y cara $(20 \%)^{22}$.

Las lesiones se inician en el sitio de contacto, con una pápula eritematosa pruriginosa de crecimiento radial en días, produciendoe lesiones anulares, eritemato-descamativas, con borde activo y múltiples vesículas ${ }^{2}$. En este tipo de tiña se presentan también dos formas principales, la microspórica y la tricofítica. La forma microspórica se encuentra conformada por múltiples placas eritemato-descamativas muy pruriginosas y son más frecuentes en los niños, ya que tienen mayor contacto con gatos y perros, mientras que la variedad tricofítica se presenta más en adultos con una lesión única y extensa ${ }^{22}$ (FIGURA 2).

\section{Tiña incógnita}

Cuando una lesión de tiña es tratada de manera crónica con esteroides tópicos, se producen las lesiones de tiña incógnita, caracterizadas por sus propiedades poco definidas, evolución tórpida y contorno irregular, las cuales son de muy difícil diagnóstico, ya que se encuentran modificadas por el tratamiento. Al suprimir el esteroide, la lesión retoma las características de la tiña ${ }^{2,50}$ (FIGURA 3). 


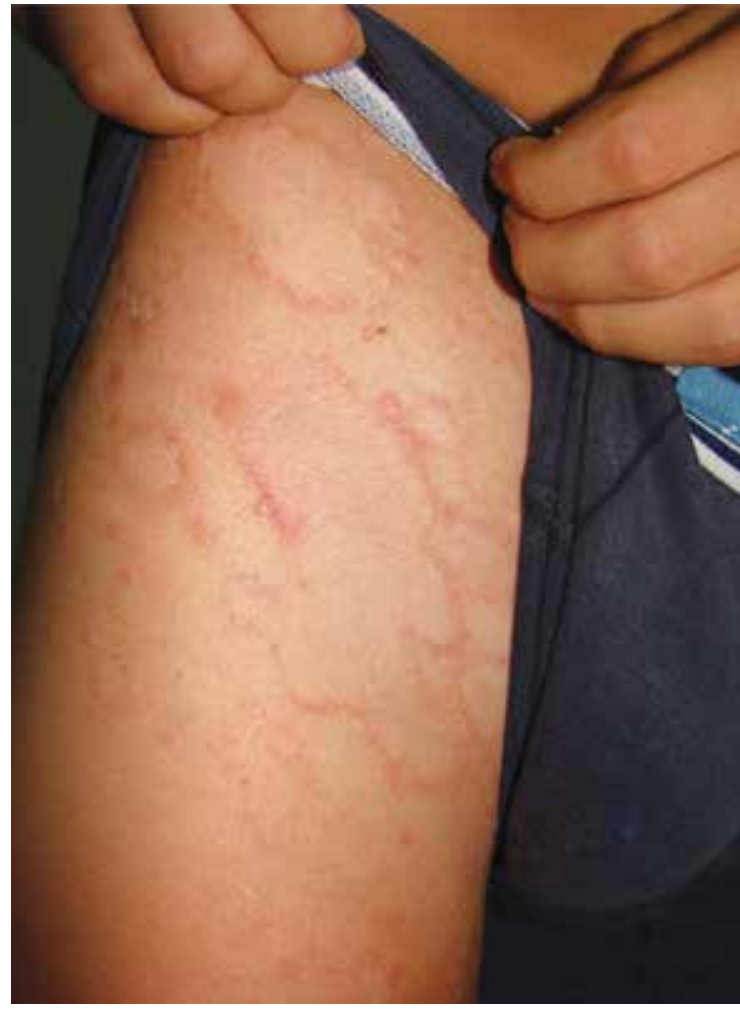

FIGURA 2. Tiña del cuerpo (tinea corporis) en un niño. Nótese el borde eritematoso y descamativo bien definido.

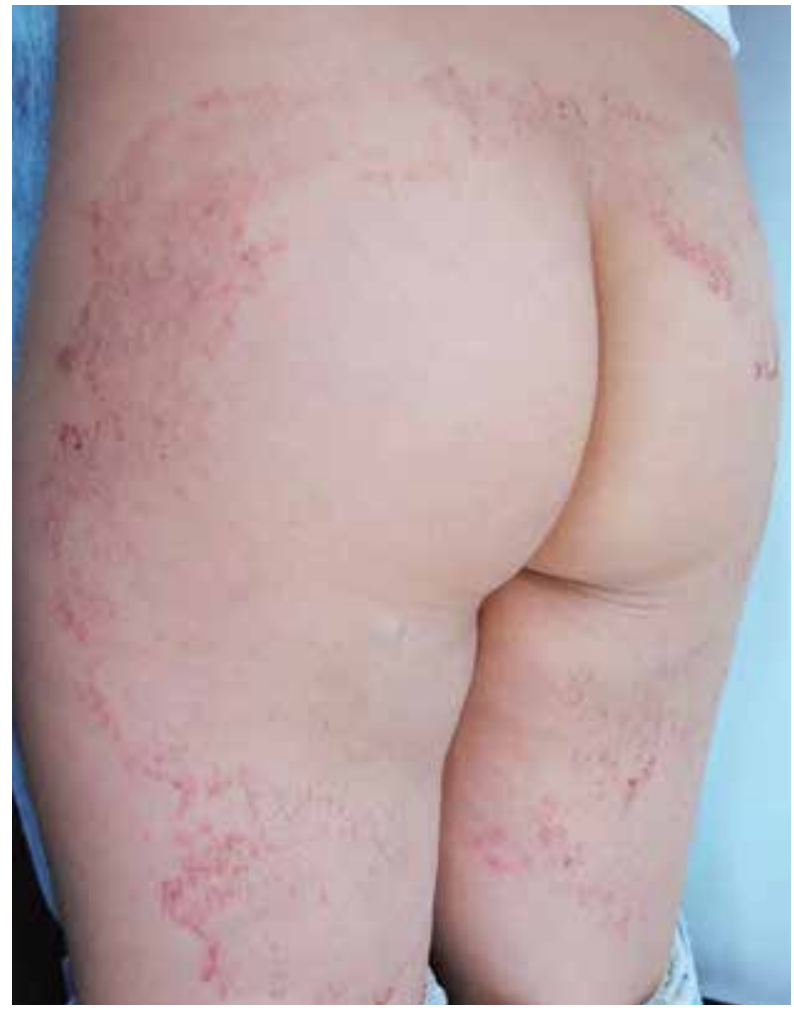

FIGURA 3. Tiña incógnita en un niño. Recibió tratamiento con múltiples esteroides tópicos.

\section{Diagnóstico diferencial}

Incluye granuloma anular, pitiriasis rosada, eccema numular, eritema anular centrífugo, lepra tuberculoide, psoriasis anular, dermatitis seborreica, dermatitis de contacto, pitiriasis versicolor y candidiasis ${ }^{22}$.

\section{Tratamiento}

Generalmente, el tratamiento de la tiña del cuerpo no conlleva mayores problemas, y el tratamiento tópico es de elección cuando las lesiones no son muy extensas y el paciente es inmunocompetente. Los azoles tópicos y las alilaminas son de utilidad y se aplican por un lapso de dos a tres semanas.

Cuando se requiere el tratamiento sistémico, pueden usarse los azoles sistémicos como el itraconazol y las alilaminas (terbinafina), por el mismo lapso de tiempo y a las mismas dosis que para la tiña de la cabeza.

\section{Tiña de las uñas (tinea unguium)}

Aunque es un padecimiento más frecuente en adultos que se encuentran más expuestos a trauma y tienen un plato ungular más grande y de crecimiento más lento ${ }^{33,51}$, cabe mencionarla brevemente, ya que en los últimos 15 años se ha visto un aumento en su incidencia en la población pediátrica, principalmente en adolescentes con edad promedio de 12 años. Aún no es posible determinar si este aumento se debe a una mayor frecuencia de la entidad o a más diagnósticos y consultas ${ }^{51-53}$.

Hasta el momento no se han encontrado diferencias en sexo o raza ${ }^{52,54}$. Los factores de riesgo son presencia de la enfermedad en los padres, uso de zapatos de plástico y cerrados, traumas deportivos, uso de piscinas y zonas húmedas, contacto con animales, inmunosupresión, diabetes mellitus y síndrome de Down ${ }^{52,55}$. Las uñas más afectadas son las de los pies y la principal forma de presentación es la subungular distal y lateral ${ }^{5}$. Los dermatofitos más aislados son en primer lugar T. rubrum, seguido por T. mentagrophytes y $T$. interdigitale $^{51,55,57,58}$. Cabe destacar que también hay 6 a $14 \%$ de portadores asintomáticos ${ }^{52,59}$.

\section{Diagnóstico}

Debe hacerse con base en la clínica y el laboratorio. Se deben demostrar las hifas en el examen directo con $\mathrm{KOH}$ o azul de clorazol E y posteriormente cultivar la muestra en agar Sabouraud, aunque con este medio 
se pueden presentar falsos positivos y se obtiene una sensibilidad de 30 a $50 \%$; en medios con antibiótico, como el Mycosel ${ }^{\mathrm{TM}}$, esta se puede aumentar hasta $79 \%$. También puede tomarse biopsia de la placa ungular para el estudio histopatológico con coloración de ácido peryódico de Schiff (PAS), obteniéndose una sensibilidad cercana al $100 \%$; no obstante, es una técnica dolorosa que en la mayoría de casos no es aceptada por el paciente ${ }^{61}$.

\section{Diagnóstico diferencial}

Debe tenerse en cuenta que en la población pediátrica puede haber otros trastornos de la uña que se confunden fácilmente con onicomicosis si no se hace un abordaje diagnóstico adecuado, como la dermatitis atópica que puede llevar a distrofia ungular, la alopecia areata que puede comprometer las uñas, la psoriasis ungular que no es infrecuente en estos pacientes, el liquen plano ungular, la enfermedad de Darier y el eccema ${ }^{22,55}$.

\section{Tratamiento}

Hasta el momento no existe una guía que indique el tratamiento en la población pediátrica e, incluso algunos autores recomiendan hacer tratamiento tópico en la mayoría de casos, pero otros refieren que si no se hace un tratamiento sistémico adecuado, la enfermedad puede pasar a otras uñas o a otros miembros de la familia, perpetuando así la infección ${ }^{53}$. La amorolfina y la ciclopiroxolamina tópicas han mostrado efectividad y los medios químicos para la avulsión de la lámina, como urea al $40 \%$ también son útiles ${ }^{62}$. Se han obtenido buenos resultados con la combinación de urea al $40 \%$ y bifonazol al $1 \%$, aunque con pocos estudios ${ }^{63}$.

En caso de múltiple afección ungular, onicomicosis distrófica total o compromiso ungular proximal, se deben considerar los tratamientos sistémicos, aunque no se encuentran aprobados por la Food and Drug Administration (FDA) para uso en niños.

En la literatura se encuentran múltiples reportes de pacientes tratados de forma exitosa con medicamentos sistémicos ${ }^{52,53,64}$. Entre las alternativas terapéuticas más usadas se encuentra el itraconazol, que puede administrarse en forma diaria o en pulsos semanales a dosis de $5 \mathrm{mg} / \mathrm{kg}$ al día, con la misma duración que en adultos ${ }^{52,64,65}$. La terbinafina presenta un mejor perfil de seguridad, siendo también útil en pulsos y en tratamiento continuo ${ }^{52,66}$. La dosis recomendada es la misma que para la tiña de la cabeza y la duración es de tres a cuatro meses ${ }^{52}$.

\section{Candidiasis cutánea superficial}

Es una infección superficial que con frecuencia se encuentra en la población general, pero predomina en pacientes con algún tipo de inmunosupresión, bien sea por el uso de inmunosupresores tópicos y sistémicos o por otras condiciones como diabetes mellitus, leucemias, linfomas, trasplantes, VIH-SIDA y en los recién nacidos, aunque también hay reportes de casos en individuos inmunocompetentes ${ }^{67}$.

Hasta el momento el agente etiológico aislado con mayor frecuencia es Candida albicans, pero se han encontrado 12 especies patógenas, con un incremento en los últimos años de C. glabratta, C. tropicalis, C. krusei, C. gulliermondii y C. parapsilosis ${ }^{67,68}$. Las especies de Candida son levaduras ovoides y algunas de ellas son saprofitos de piel y mucosas ${ }^{67}$.

La población pediátrica está expuesta, ya que la colonización se inicia desde el primer día del nacimiento y persiste de por vida, tornándose infecciosa sólo en condiciones de inmunosupresión local o sistémica. Las principales formas de presentación son intertrigo candidiásico, miliaria candidiásica, queilitis angular o perleche y candidiasis oral o muguet ${ }^{2}$.

\section{Intertrigo candidiásico}

Es una lesión húmeda que se inicia en los pliegues donde la fricción, el calor y la obesidad maceran la piel, permitiendo la entrada de esta levadura. Se encuentra principalmente en las regiones axilar, inguinal, submamaria e interglútea. El cuadro inicia con vesículas pequeñas sobre una base eritematosa, que posteriormente confluyen formando una placa de color rojo, con bordes descamativos y lesiones satélite ${ }^{2,68}$. En recién nacidos y lactantes, esta forma se presenta en el área del pañal, por colonización proveniente del sistema gastrointestinal ${ }^{67}$.

\section{Miliaria candidiásica}

Resulta de la colonización de lesiones de miliaria; son vesículo-pústulas descamativas y pruriginosas, principalmente en la espalda ${ }^{69}$.

\section{Candidiasis oral o muguet}

Consiste en lesiones ubicadas en la cavidad oral, que se inician como máculas eritematosas y posteriormente adquieren una superficie untuosa blanquecina de aspecto cremoso que, al intentar removerla, deja una mucosa hiperémica².

\section{Queilitis angular o perleche}

Se presentan fisuras, maceración y eritema en los ángulos de la boca ${ }^{70}$. 


\section{Candidiasis}

\section{crónica mucocutánea}

La candidiasis crónica mucocutánea comprende un grupo de alteraciones heterogéneas caracterizadas por infecciones persistentes no invasivas y en raras ocasiones sistémicas ${ }^{67}$, afectando uñas, piel y membranas mucosas, por diferentes especies de Candida ${ }^{71}$. Esta entidad es causada por una inmunodeficiencia primaria, por lo que se manifiesta desde temprana edad y su patogénesis implica una respuesta inmunitaria inefectiva por defectos en varios de los procesos, en particular en las células T ayudadoras ( $T$ helper) de tipo Th17, que son las principalmente implicadas en la defensa del huésped contra estas levaduras ${ }^{71}$. Se han reportado mutaciones genéticas en proteínas cruciales para crear una defensa inmunitaria efectiva, particularmente en el transductor de señal y activador de transcripción 1 (STAT1) ${ }^{71}$.

Esta entidad se encuentra también asociada a trastornos endocrinos, como hipotiroidismo, enfermedad de Addison, diabetes mellitus, hipogonadismo y hepatitis autoinmunitaria ${ }^{67772}$.

\begin{tabular}{|c|c|c|c|}
\hline Entidad & Pitiriasis versicolor & Piedra blanca & Tiña negra \\
\hline Etiología & $\begin{array}{l}\text { Malassezia (M. furfur, } \\
\text { symphodyalis, globosa) }\end{array}$ & Trichosporon (T. inkin,ovoide) $)^{76}$ & Hortaea werneckii \\
\hline Frecuencia & $20 \%$ & Rara & Rara \\
\hline Edad más frecuente & 14 a 18 años & 2 a 6 años ${ }^{77-79}$ & 2 a 18 años \\
\hline $\begin{array}{l}\text { Factores } \\
\text { predisponentes }\end{array}$ & $\begin{array}{l}\text { Genética, humedad local, } \\
\text { pubertad y adolescencia, } \\
\text { uso de esteroides, estados } \\
\text { carenciales, calor } 2,75,80\end{array}$ & $\begin{array}{l}\text { Climas cálidos, recoger el pelo } \\
\text { húmedo, uso de aceites vegetales } 77\end{array}$ & $\begin{array}{l}\text { Zonas costeras e hipersalinas, } \\
\text { hiperhidrosis, trauma superficial, } \\
\text { contacto con pasto }\end{array}$ \\
\hline Clínica & $\begin{array}{l}\text { Placas hipopigmentadas } \\
\text { o hiperpigmentadas en } \\
\text { áreas seborreicas (tronco, } \\
\text { brazos, cuello, cara) }{ }^{80} \text { Prurito } \\
\text { ocasional }\end{array}$ & $\begin{array}{l}\text { Nódulos blanco-amarillos, } 1 \text { a } 2 \\
\text { mm, firmemente adheridos, suaves } \\
\text { (cuero cabelludo, axilas, pubis). } \\
\text { Sin invasión endothrix. Debilita y } \\
\text { fractura el pelo. Asintomáticos } 77,79\end{array}$ & 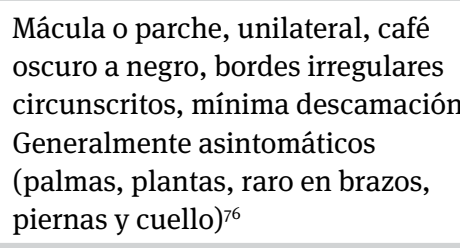 \\
\hline Diagnóstico diferencial & $\begin{array}{l}\text { Pitiriasis alba, pitiriasis } \\
\text { rosada, pigmentación } \\
\text { posinflamatoria, vitiligo, tiña } \\
\text { del cuerpo, eritrasma }{ }^{76}\end{array}$ & $\begin{array}{l}\text { Pediculosis, moniletrix, } \\
\text { tricoptilosis, tricorrexis nudosa }\end{array}$ & $\begin{array}{l}\text { Melanoma lentiginoso acral } \\
\text { incipiente, pigmentación } \\
\text { posinflamatoria, nevus de unión }{ }^{82}\end{array}$ \\
\hline Diagnóstico & $\begin{array}{l}\text { Clínico (rascado, luz de Wood) } \\
\text { y por laboratorio. } \\
\text { KOH: imagen en espaguetti } \\
\text { con albóndigas }^{80}\end{array}$ & $\begin{array}{l}\text { Por laboratorio. } \mathrm{KOH} \text { : nódulos } \\
\text { que rodean el pelo, filamentos } \\
\text { tabicados } 2 \text { a } 4 \text { micras entre } \\
\text { las células de la cutícula y } \\
\text { artroconidias ovaladas o redondas. } \\
\text { Cultivo: colonias blancas, lisas, } \\
\text { crecimiento } 10 \text { a } 12 \text { días, aspecto } \\
\text { cerebriforme posteriormente }\end{array}$ & $\begin{array}{l}\text { Por clínica y laboratorio. } \\
\text { Dermatoscopia: mácula sin } \\
\text { red de pigmento café o negro; } \\
\text { examen directo con } \mathrm{KOH} \text { : hifas } \\
\text { septadas pigmentadas con células } \\
\text { levaduriformes agrupadas; } \\
\text { cultivo: colonias cremosas de color } \\
\text { oscuro }^{76}\end{array}$ \\
\hline Tratamiento & $\begin{array}{l}\text { Tópico: sulfuro de selenio, } \\
\text { ketoconazol, piritionato } \\
\text { de cinc, ácido salicílico, } \\
\text { azoles en champú o jabón. } \\
\text { Terbinafina al } 1 \text { \% (dos } \\
\text { semanas). En casos extensos } \\
\text { o resistentes, ketoconazol } \\
\text { sistémico, } 200 \text { mg/día por dos } \\
\text { semanas; otros: fluconazol y } \\
\text { itraconazol }\end{array}$ & $\begin{array}{l}\text { Corte de pelo } \\
\text { Antifúngicos tópicos } \\
\text { Queratolíticos (ácido salicílico) } \\
\text { Sistémico solo en infecciones } \\
\text { extensas o que no mejoren con } \\
\text { tratamiento tópico (terbinafina, } \\
\text { itraconazol, fluconazol) }\end{array}$ & $\begin{array}{l}\text { Controlar condiciones de base. } \\
\text { Queratolíticos (ácido salicílico al } \\
3 \% \text {, úrea, solución de Whitfield) } \\
\text { Antifúngicos tópicos } \\
\text { Lesiones persistentes: itraconazol } \\
\text { oral } 100 \mathrm{mg} / \mathrm{dia}^{76,81}\end{array}$ \\
\hline Pronóstico & Crónica y recurrente & Recurrente & Recurrente \\
\hline
\end{tabular}

TABLA 1. Otras entidades frecuentes en la infancia. 


\section{Diagnóstico}

En el examen directo con $\mathrm{KOH}$, negro de clorazol, calcoflúor o coloración de Gram, se observan blastoconidias ovaladas de pared sencilla y con formación de seudomicelios.

En el cultivo se observa crecimiento de colonias blancas o amarillas cremosas, con crecimiento en 48 a 72 horas a $37^{\circ} \mathrm{C}$. Se requieren pruebas bioquímicas y estudios morfológicos, como medios de cultivo cromógenos, para identificar cada especie ${ }^{22}$.

\section{Diagnóstico diferencial}

Para el intertrigo candidiásico deben tenerse en cuenta el eritrasma, la psoriasis inversa y la dermatitis seborreica; en el muguet debe considerarse en general cualquier tipo de estomatitis ${ }^{2}$, y para el perleche se deben tener en cuenta los subtipos de queilitis.

\section{Tratamiento}

El principal abordaje en el tratamiento de la candidiasis es el control de la enfermedad de base, ya que este es el principal factor implicado en el desarrollo de la infección. Se debe controlar la humedad, el calor, la fricción y las enfermedades que predisponen a esta en$\operatorname{tidad}^{67}$. Para la candidiasis cutánea los antifúngicos tópicos son los más usados, principalmente los imidazoles y alilaminas, en intervalos cada 12 horas y hasta 15 días después de la resolución clínica; en caso de persistencia de las lesiones, se debe considerar el tratamiento sistémico ${ }^{73}$. En cuanto a la candidiasis oral, el tratamiento más usado es la nistatina en suspensión, que se suministra en forma de enjuagues cada seis a ocho horas por una a dos semanas, ya que tienen un buen cubrimiento frente a Candida y no tienen absorción gastrointestinal ${ }^{70}$.

Además de las entidades anteriormente mencionadas, deben tenerse en cuenta otras micosis superficiales presentes en la infancia, que, aunque no son tan frecuentes, pueden encontrarse en las consultas dermatológicas (TABLA 1).

\section{Conclusión}

Las micosis en la infancia, aunque no son las entidades más comunes, tienen una frecuencia considerable y una morbilidad importante, por lo cual se deben tener presentes a la hora de evaluar al niño con síntomas. Esto es así, principalmente, en las infecciones por dermatofitos, ya que son las más importantes en dicho grupo etario, pero sin dejar atrás las demás micosis que en ocasiones, por su baja frecuencia, pueden pasar inad- vertidas y llevar a fallas en el tratamiento y resultados desfavorables para el paciente.

\section{Referencias}

1. Ruiz A, Ciroco A. Micosis cutáneas en la Infancia : estudio retrospectivo desde 1990 hasta el 2001 en la consulta de micología del Servicio de Dermatología del Hospital Universitario de Caracas. Dermatol Venez. 2004;42(1):30-4.

2. Rojo J, González C. Micosis cutáneas en los niños. Pediatr Integr. 2004;8(4):271-8.

3. Sellami A, Sellami H, Makni F, Mezghani S, Cheikh-Rouhou F, Marrekchi S, et al. Childhood dermatomycoses study in Sfax Hospital, Tunisia. Mycoses. 2008 Sep;51(5):451-4.

4. Fernandes NC, Akiti T, Barreiros MG. Dermatophytoses in children: study of 137 cases. Rev. Inst. Med. trop. S. Paulo. 2001;43(2):83-5.

5. Seidl HP, Ring J, Abeck D. Pediatric Tinea Capitis: recognition and management. Am J Clin Dermatol. 2005;6(4):203-13.

6. Rebollo N, López-Barcenas AP, Arenas R. Tiña de la cabeza. Actas Dermosifiliogr. 2008 Mar;99(2):91-100.

7. Emele FE, Oyeka $\mathrm{C}$ a. Tinea capitis among primary school children in Anambra state of Nigeria. Mycoses. 2008 Nov;51(6):536-41.

8. Aly R. Ecology, epidemiology and diagnosis of tinea capitis. Pediatr. Infect. Dis. J. 1999 Feb;18(2):180-5.

9. Fuller LC, Child FC, Higgins EM. Tinea capitis in south-east London: an outbreak of Trichophyton tonsurans infection. Br. J. Dermatol. 1997 Jan;136(1):139.

10. Del Boz J, Crespo V, Rivas-Ruiz F, de Troya M. A 30-year survey of paediatric tinea capitis in southern Spain. J. Eur. Acad. Dermatol. Venereol. 2011 Feb;25(2):170-4.

11. Ali S, Graham TAD, Forgie SED. The assessment and management of tinea capitis in children. Pediatr. Emerg. Care. 2007 Sep;23(9):662-5; quiz 666-8.

12. Fuller LC, Child FJ, Midgley G, Higgins EM. Diagnosis and management of scalp ringworm. BMJ. 2003 Mar 8;326(7388):539-41.

13. Ginter-Hanselmayer G, Weger W, Ilkit M, Smolle J. Epidemiology of tinea capitis in Europe: current state and changing patterns. Mycoses. 2007 Jan;50 Suppl 2:6-13.

14. Benavides J, Villanueva J. Tiña del cuero cabelludo por Trichophyton tonsurans: agente emergente en Colombia. Rev Asoc Colomb Dermatol. 2010;18:99-101.

15. Santa Maria L, Vélez H, Guzmán G. DERMATOMICOSIS ANALISIS RETROSPECTIVO DE 2.336 PACIENTES, 1976-1980 [Internet]. [cited 2014 Aug 11]. Available from: http://www.actamedicacolombiana. com/anexo/articulos/06-1982-03.htm

16. Elewski BE. Tinea capitis: a current perspective. J. Am. Acad. Dermatol. 2000 Jan;42(1 Pt 1):1-20; quiz 21-4.

17. Triviño-Duran L, Torres-Rodriguez JM, Martinez-Roig A, Cortina C, Belver V, Perez-Gonzalez M, et al. Prevalence of tinea capitis and tinea pedis in Barcelona school children. Pediatr. Infect. Dis. J. 2005 Feb;24(2):137-41.

18. Marples MJ. The ecology of Microsporum canis bodin in New Zeland. J Hyg. 1956;54(3):378-87.

19. Brasch J, Gottkehaskamp D. The effect of selected human steroid hormones upon the growth of dermatophytes with different adaptation to man. Mycopathologia. 1992 Nov;120(2):87-92. 
20. Pereiro Miguens M, Pereiro M. Review of dermatophytoses in Galicia from 1951 to 1987 , and comparison with other areas of Spain. Mycopathologia. 1991 Feb;113(2):65-78.

21. Gilaberte Y, Rezusta A, Gil J, Sáenz-Santamaría MC, Coscojuela C, Navarro M, et al. Tinea capitis in infants in their first year of life. Br. J. Dermatol. 2004 Oct;151(4):886-90.

22. Bonifaz A. Micología Médica Básica. 4th ed. México, DF: Mc GrawHill; 2013. p. 100-32.

23. Chanussot C, Arenas R. querion de celso por M gypseum en niño de 5 años.pdf. Dermatol Venez. 2010;48(3):99-101.

24. Schwartz RA, Janniger CK. Tinea capitis. Cutis. 1995 Jan;55(1):29-33.

25. Gupta AK, Katz HI, Shear NH. Drug interactions with itraconazole, fluconazole, and terbinafine and their management. J. Am. Acad. Dermatol. 1999 Aug;41(2 Pt 1):237-49.

26. Bojórquez MA, Arenas R, Molina de Soschin D V-ME. Granuloma tricofítico y querión de Celso: Datos clínicos e histológicos de nueve casos. Dermatologia Rev Mexo2. 2002;46:15-22.

27. Honig PJ, Caputo GL, Leyden JJ, McGinley K, Selbst SM, McGravey AR. Microbiology of kerions. J. Pediatr. 1993 Sep;123(3):422-4.

28. Babel DE, Baughman SA. Evaluation of the adult carrier state in juvenile tinea capitis caused by Trichophyton tonsurans. J. Am. Acad. Dermatol. 1989 Dec;21(6):1209-12.

29. Hebert AA. Tinea capitis. Current concepts. Arch. Dermatol. 1988 Oct;124(10):1554-7.

30. Viguié-Vallanet C, Serre M, Masliah L, Tourte-Schaefer C. [Epidemic of Trichophyton tonsurans tinea capitis in a nursery school in the Southern suburbs of Paris]. Ann. Dermatol. Venereol. 2005 May;132(5):432-8.

31. Sharma V, Hall JC, Knapp JF, Sarai S, Galloway D, Babel DE. Scalp colonization by Trichophyton tonsurans in an urban pediatric clinic. Asymptomatic carrier state. Arch. Dermatol. 1988 Oct;124(10):1511-3.

32. Kefalidou S, Odia S, Gruseck E, Schmidt T, Ring J, Abeck D. Wood's light in Microsporum canis positive patients. Mycoses. 1997 Dec;40(11-12):461-3.

33. Elewski BE. Cutaneous mycoses in children. Br. J. Dermatol. 1996 Jun;134 Suppl:7-11: discussion 37-8.

34. Higgins EM, Fuller LC, Smith CH. Guidelines for the management of tinea capitis. British Association of Dermatologists. Br. J. Dermatol. 2000 Jul;143(1):53-8.

35. Arenas R, Toussaint S, Isa-Isa R. Kerion and dermatophytic granuloma. Mycological and histopathological findings in 19 children with inflammatory tinea capitis of the scalp. Int. J. Dermatol. 2006 Mar;45(3):215-9.

36. Sperling LC. Inflammatory tinea capitis (kerion) mimicking dissecting cellulitis. Occurrence in two adolescents. Int J Dermatol. 1991 Mar;30(3):190-2.

37. Hussain I, Muzaffar F, Rashid T, Ahmad TJ, Jahangir M, Haroon TS. A randomized, comparative trial of treatment of kerion celsi with griseofulvin plus oral prednisolone vs. griseofulvin alone. Med. Mycol. 1999 Apr;37(2):97-9.

38. Allen HB, Honig PJ, Leyden JJ, McGinley KJ. Selenium sulfide: adjunctive therapy for tinea capitis. Pediatrics. 1982 Jan;69(1):81-3.

39. Neil G, Hanslo D, Buccimazza S, Kibel M. Control of the carrier state of scalp dermatophytes. Pediatr. Infect. Dis. J. 1990 Jan;9(1):57-8.

40. González U, Seaton T, Bergus G, Jacobson J, Martínez-Monzón C. Systemic antifungal therapy for tinea capitis in children. Cochrane database Syst. Rev. 2007 Jan;(4):CDoo4685.
41. Moossavi M, Bagheri B, Scher RK. Systemic antifungal therapy. Dermatol. Clin. 2001 Jan;19(1):35-52.

42. Krafchik B, Pelletier J. An open study of tinea capitis in 50 children treated with a 2-week course of oral terbinafine. J Am Acad Dermatol. 1999 Jul;41(1):60-3.

43. Koumantaki E, Kakourou T, Rallis E, Riga P, Georgalla S. Doubled dose of oral terbinafine is required for Microsporum canis tinea capitis. Pediatr. Dermatol. 2001;18(4):339-42.

44. Nejjam F, Zagula M, Cabiac MD, Guessous N, Humbert H, Lakhdar H. Pilot study of terbinafine in children suffering from tinea capitis: evaluation of efficacy, safety and pharmacokinetics. Br. J. Dermatol. 1995 Jan;132(1):98-105.

45. Jones TC. Overview of the use of terbinafine (Lamisil) in children. Br. J. Dermatol. 1995 May;132(5):683-9.

46. Conjeevaram G, Vongthavaravat V, Sumner R, Koff RS. Terbinafine-induced hepatitis and pancytopenia. Dig. Dis. Sci. 2001 Aug;46(8):1714-6.

47. Abdel-Rahman SM, Powell DA, Nahata MC. Efficacy of itraconazole in children with Trichophyton tonsurans tinea capitis. J. Am. Acad. Dermatol. 1998 Mar;38(3):443-6.

48. Haria M, Bryson HM, Goa KL. Itraconazole. A reappraisal of its pharmacological properties and therapeutic use in the management of superficial fungal infections. Drugs. 1996 Apr;51(4):585-620.

49. Niewerth M, Korting HC. The use of systemic antimycotics in dermatotherapy. Eur. J. Dermatol. 2000 Mar;10(2):155-60.

50. Kawakami Y, Oyama N, Sakai E, Nishiyama K, Suzutani T, Yamamoto T. Childhood Tinea incognito caused by Trichophyton mentagrophytes Var. Interdigitale mimicking pustular psoriasis. Pediatr. Dermatol. 2011;28(6):738-40.

51. Sigurgeirsson B, Kristinsson KG, Jonasson PS. Onychomycosis in Icelandic children. JEADV. 2006 Aug;20(7):796-9.

52. Vásquez del Mercado E, Arenas R. Onicomicosis en niños. Estudio retrospectivo de 233 casos mexicanos. Gac Méd Méx. 2008;144(1):7-10.

53. Gupta AK, Skinner AR, Baran R. Onychomycosis in children: an overview. J. Drugs Dermatol. 2003 Jan;2(1):31-4.

54. Lange M, Nowicki R, Bara Dska-Rybak W, Bykowska B. Dermatophytosis in children and adolescents in Gdansk, Poland. Mycoses. 2004 Aug;47(7):326-9.

55. Romano C, Papini M, Ghilardi A, Gianni C. Onychomycosis in children: a survey of 46 cases. Mycoses. 2005 Nov;48(6):430-7.

56. Gupta AK, Skinner AR. Onychomycosis in children: a brief overview with treatment strategies. Pediatr. Dermatol. 2004;21(1):74-9.

57. Arenas R. [Onychomycosis. Clinico-epidemiological mycological and therapeutic aspects]. Gac. Med. Mex. 1990;126(2):84-9; discussion 90-1.

58. Ginter-Hanselmayer G, Weger W, Smolle J. Onychomycosis: a new emerging infectious disease in childhood population and adolescents. Report on treatment experience with terbinafine and itraconazole in 36 patients. JEADV. 2008 Apr;22(4):470-5.

59. Becerril-Chihu G, Bazán-Mora E, López-Martínez R, Sosa-de-Martínez C, Ruiz-Maldonado R. How often are dermatophytes present in apparently normal versus scaly feet of children? Pediatr. Dermatol. 1999;16(2):87-9.

6o. Villanueva J, Arenas R. Candidiasis mucocutánea. Una revisión. Rev Méx Micol. 2007;25:91-104.

61. Gupta AK, Tu LQ. Onychomycosis therapies: strategies to improve efficacy. Dermatol. Clin. 2006 Jul;24(3):381-6. 
62. Alam M, Scher RK. Current topics in nail surgery. J. Cutan. Med. Surg. 1999 Oct;3(6):324-35.

63. Bonifaz A, Ibarra G. Onychomycosis in children: treatment with bifonazole-urea. Pediatr. Dermatol. 2000;17(4):310-4.

64. Tosti A, Piraccini BM, Iorizzo M. Management of onychomycosis in children. Dermatol. Clin. 2003 Jul;21(3):507-9, vii.

65. Gupta AK, Cooper EA, Ginter G. Efficacy and safety of itraconazole use in children. Dermatol. Clin. 2003 Jul;21(3):521-35.

66. Baran R, Hay RJ, Garduno JI. Review of antifungal therapy, part II: treatment rationale, including specific patient populations. J. Dermatolog. Treat. 2008 Jan;19(3):168-75.

67. Palacios CP, Gómez LM, Cardona N. Candidiasis mucocutánea : espectro clínico. Rev Asoc Colomb Dermatol. 2011;19:239-44.

68. López-Martínez R. Candidosis, a new challenge. Clin. Dermatol. 2010 Mar 4;28(2):178-84.

69. Sullivan DJ, Westerneng TJ, Haynes KA, Bennett DE, Coleman DC. Candida dubliniensis sp. nov.: phenotypic and molecular characterization of a novel species associated with oral candidosis in HIV-infected individuals. Microbiology. 1995;141:1507-21.

70. Farah C, Ashman R, Challacombe S. Oral candidosis. Clin. Dermatol. 2000;18(5):553-62.

71. Rushood M, McCusker C, Mazer B, Alizadehfar R, Grimbacher B, Depner M. Autosomal Dominant Cases of Chronic Mucocutaneous Candidiasis Segregates with Mutations of Signal Transducer and Activator of Transcription 1, But Not of Toll-Like Receptor 3. J Pediatr. 2013;163:277-9.

72. Lilic D, Gravenor I, Robson N, Lammas DA, Drysdale P, Calvert JE, et al. Deregulated Production of Protective Cytokines in Response to Candida albicans Infection in Patients with Chronic Mucocutaneous Candidiasis. Infect. Immun. 2003;71(10):5690-9.
73. Chen SCA, Sorrell TC. Antifungal agents. Med. J. Aust. 2007 Oct 1;187(7):404-9.

74. Park HJ, Lee YW, Choe YB, Ahn KJ. Skin Characteristics in Patients with Pityriasis Versicolor Using Non-Invasive Method, MPA5. Ann. Dermatol. 2012 Nov;24(4):444-52.

75. Bonifaz A, Gómez-Daza F, Paredes V, Ponce RM. Tinea versicolor, tinea nigra, white piedra, and black piedra. Clin. Dermatol. Elsevier Inc.; 2010 Mar 4;28(2):140-5.

76. Ríos X, Rojas R, Hincapié ML. Ocho casos de piedra blanca en población pediátrica. Rev Asoc Colomb Dermatol. 2012;20(2):175-80.

77. Roshan AS, Janaki C, Parveen B. White piedra in a mother and daughter. Int. J. Trichology. 2009 Jul;1(2):140-1.

78. Muñoz VF, Díaz EE, González JL, Trejo JR. Piedra blanca en una paciente pediátrica: reporte de un caso. Rev Iberoam Micol. 2009;26(4):252-4.

79. Padilla M del C. Pitiriasis versicolor. Dermatología Rev Mex. 2005;49:157-67.

8o. Bonifaz A, Badali H, de Hoog GS, Cruz M, Araiza J, Cruz M a, et al. Tinea nigra by Hortaea werneckii, a report of 22 cases from Mexico. Stud. Mycol. 2008 Jan;61:77-82.

81. Tilak R, Sinigh S, Prakash P, Singh D, Gulati A. A case report for tinea nigra in north India. Indian J Dermatol Venereol Leprol. 2009;75(5):538-9.

82. Khandpur S, Reddy BS. Itraconazole therapy for white piedra affecting scalp hair. J. Am. Acad. Dermatol. 2002 Sep;47(3):415-8. 